International Journal of Bioinformatics Research

ISSN: 0975-3087, E-ISSN: 0975-9115, Vol. 3, Issue 1, 2011, pp-194-199

Available online at http://www.bioinfo.in/contents.php?id=21

\title{
PSEUDOKNOTS IN HUMAN SNRNPS
}

\section{SANJAY KUMAR DEY, SAYAK GANGULI*, PAUSHALI ROY, PROTIP BASU, HIRAK JYOTI CHAKRABORTY AND ABHIJIT DATTA ${ }^{1}$}

*DBT-Centre for Bioinformatics, Presidency University, Kolkata

1Post Graduate Department of Botany, Bethune College, Kolkata

*Corresponding author. E-mail: sayakbif@yahoo.com

Received: April 05, 2011; Accepted: May 20, 2011

\begin{abstract}
Snurps or small nuclear ribonucleoproteins (snRNPs), are RNA-protein complexes that combine with unmodified pre-mRNA and various other proteins to form a Spliceosome, comprising of five small nuclear RNAs (snRNAs)-U1, U2, U4, U5, and U6 snRNA-as well as many protein factors, upon which splicing of pre-mRNA occurs. While, RNA pseudoknots play crucial role in protein synthesis by helping in internal ribosome entry, frameshifting, stop codon readthrough in many viral species and the 3'NCR pseudoknots helps viral RNAs to replicate, has been reported by a number of investigators, its presence in human snurps has not yet been done. The present in silico study reveals the presence of pseudoknots in the mRNAs of the proteins associated with human Spliceosome. It not only emphasizes their significance as catalytic RNA world relics but also opens the scope of research in the functional and structural associations of RNA pseudoknots in eukaryotic gene regulation.
\end{abstract}

Keywords: snRNP, splicing, pseudoknot, gene regulation, catalytic RNA world.

\section{Introduction}

Small nuclear ribonucleoproteins (snRNPs) are known to assemble in a stepwise manner onto the pre-mRNA to form the spliceosome. The spliceosome is comprised of five small nuclear RNAs (snRNAs)—U1, $\mathrm{U} 2, \mathrm{U} 4, \mathrm{U} 5$, and $\mathrm{U} 6 \mathrm{SnRNA}$ and many protein factors $[1,2]$. The coding regions (exons) of the gene are separated by non-coding DNA (introns) that are not involved in gene expression. These introns are removed by the process known as splicing, resulting in the finally processed mRNA. An RNA pseudoknot is a tertiary structural element formed when bases of a single- stranded loop pair interact with complementary bases outside the loop within the RNA molecule $[3,4]$. Though the presences of 14 topologically possible distinct types of pseudoknots have been hypothesized, most commonly occurring pseudoknots are of the H-type, where $\mathrm{H}$ signifies a hairpin loop [3]. The other important types are HH type, LL type, HLout type and HLIN type etc. [3]. In the case of H-type pseudoknots, bases in a hairpin loop pair with complementary bases outside the loop (Fig.1). HH-type pseudoknot is formed by base pairing of a hairpin loop with another haimin loop and $\mathrm{HL}$ type pseudoknot is formed due to base pairing of a hairpin loop with a single stranded part of a bulge or of an internal or multiple loops (Fig.1). Base pairing of single stranded bulge (B), interior (I) and multibranched (M) loops with complementary regions elsewhere in the RNA also makes pseudoknots. The $\mathrm{B}, \mathrm{M}$ and I nomenclatures as well as $\mathrm{H}-\mathrm{H}$ type are not used extensively; rather most structures are referred to as H-type pseudoknots [4].
In eukaryotes, the pseudoknots in $80 \mathrm{~S}$ ribosomal complex correspond to an $L$ shaped density located at the mRNA exit channel of ribosomal proteins S5 (rsS5). These pseudoknots are important for the formation of the ribosomal A (amino-acyl), P (peptidyl) and $E$ (exit) sites by the interaction of the IRES (internal ribosome entry site) with many key protein component and the $80 \mathrm{~S}$ complex during protein synthesis [4].

Programmed-ribosomal Frameshifting is a translational mechanism used by many viruses to coordinately express proteins from an mRNA [5]. RNA pseudoknots within the coding regions are mainly responsible for this frame shifting activity. Another important function of the pseudoknot is to facilitate read through of the stop codon, in this regard H-type pseudoknot present at eight nucleotides downstream of the stop codon has been reported to enhance the process [6]. Pseudoknots are also used as binding sites for proteins or single stranded loops of RNA. Indeed pseudoknotted RNAs are often generated during in vitro selection of RNA aptamers that bind various biomolecules [4]. Pseudoknotting is also being the most efficient way of folding RNAs in an active conformation (for example- ribozymes) [4].

The role of RNA pseudoknots in translation has been reported in detail, but its presence in human snurps has yet not been revealed. Thus identification of RNA pseudoknots in the mRNAs of the protein part of the human snRNPs will be of excellent biological significance to establish the structural and functional 
relationship between the RNA pseudoknots and gene expression strategies in eukaryotes.

\section{Materials and Method}

- The DNA sequences for the human snRNPs were downloaded from the Genbank of NCBI website. The sequences used in this study are listed in Table1.

- The DNA sequences were then converted into RNA sequences using an in house tool.

- $\quad$ The RNAs were then analyzed by Pseudoviewer package.

\section{Results}

The results of the pseudoknot analysis showed four types of pseudoknots, namely, HLout, LL, classical H and HLIN types. The HLout type pseudoknot was found to be the most predominant type occurring in 41 of the cases. While $\mathrm{LL}$, classical $\mathrm{H}$ and $\mathrm{HL}$ In types of pseudoknots were found 21, 14 and 12 times respectively (Table2, Fig.1).

\section{Discussion and Conclusion}

As is evident from the results, several pseudoknot motifs were identified in the human snurps (Table2, Fig.1). The presence of this important structural element is a significant finding in RNA biology as it has been known over the years that pseudoknots are important ubiquitous structures in the mRNA which guides the process of translation through its twists and turns. Furthermore Cech [7] has emphasized that pseudoknot and associated structures formed the core of RNA based gene regulation in the ancient RNA world before the adaptive radiation of proteins in their diverse biological functions. The basics of RNA based gene regulation that exists in the modern RNA world comprises of the riboswitches and the various attenuator and repressor systems found in bacteria and few eukaryotes. The identification and characterization of pseudoknots in the proteins associated with the spliceosome provides further evidences that these pseudoknots are RNA world relics and probably originate from an ancestral stock of RNA regulons. It is important to note that the identification of RNA based regulation of important mammalian gene regulatory systems promotes the fact that we are still under the control of RNA, though there has been the evolution of DNA based genomes.

\section{Acknowledgement}

The authors acknowledge the support from Department of Biotechnology, Govt. of India for this work.

\section{References}

[1] Kruger K., Grabowski P.J., Zaug A.J., Sands J., Gottschling D.E. and Cech T.R. (1982) Cell, 31(1),147-57.

[2] Guerrier-Takada C., Gardiner K., Marsh T., Pace N. and Altman S. (1983) Cell, 35, 849-857.

[3] Brierley I., Pennell S. and Gilbert R. J. C. (2007) Nature Reviews Microbiology, 5, 598610.

[4] Namy O., Moran S. J., Stuart D. I., Gilbert R. J. C. and Brierley I. (2006) Nature, 44, 244247.

[5] Gluick T. C., Wills N. M, Gesteland R. F. and Draper D. E. (1997) Biochemistry, 36 (51),16173-16186.

[6] Gesteland R.F., Cech T.R., Atkins J.F. (eds). (2006) The RNA world (Cold Spring Harbor Laboratory, Cold Spring Harbor) 3rd edition, 309. 
Table 1- The accession numbers for DNA sequences of the human SnRNPs mRNA under study

\begin{tabular}{|c|c|c|}
\hline $\begin{array}{c}\text { Serial } \\
\text { Number }\end{array}$ & Accession Number & Description \\
\hline 1 & $>$ gi|40217846 & ref|NM_014014.2| Homo sapiens small nuclear ribonucleoprotein 200kDa (U5) (SNRNP200), mRNA \\
\hline 2 & $>$ gi|31077202 & $\begin{array}{l}\text { ref|NM_022717.2| Homo sapiens small nuclear ribonucleoprotein 35kDa (U11/U12) (SNRNP35), transcript } \\
\text { variant 2, mRNA }\end{array}$ \\
\hline 3 & $>$ gi|212549559 & $\begin{array}{l}\text { ref|NM_180703.2| Homo sapiens small nuclear ribonucleoprotein 35kDa (U11/U12) (SNRNP35), transcript } \\
\text { variant 4, mRNA }\end{array}$ \\
\hline 4 & $>$ gi|57634537 & ref|NM_003089.4| Homo sapiens small nuclear ribonucleoprotein 70kDa (U1) (SNRNP70), mRNA \\
\hline 5 & >gi|38149990 & $\begin{array}{l}\text { ref|NM_003091.3| Homo sapiens small nuclear ribonucleoprotein polypeptides B and B1 (SNRPB), transcript } \\
\text { variant 2, mRNA }\end{array}$ \\
\hline 6 & >gi|61098048 & ref|NM_003094.2| Homo sapiens small nuclear ribonucleoprotein polypeptide E (SNRPE), mRNA \\
\hline 7 & $>$ gi|29540552 & $\begin{array}{l}\text { ref|NM_022805.2| Homo sapiens small nuclear ribonucleoprotein polypeptide N (SNRPN), transcript variant 2, } \\
\text { mRNA }\end{array}$ \\
\hline 8 & $>$ gi|29540554 & $\begin{array}{l}\text { ref|NM_022807.2| Homo sapiens small nuclear ribonucleoprotein polypeptide N (SNRPN), transcript variant 4, } \\
\text { mRNA }\end{array}$ \\
\hline 9 & $>$ gi|4507126 & ref|NM_003093.1| Homo sapiens small nuclear ribonucleoprotein polypeptide C (SNRPC), mRNA \\
\hline 10 & >gi|24474790 & $\begin{array}{l}\text { emb|AJ505017.1| Homo sapiens mRNA for U5 small nuclear ribonucleoprotein component (SNRP116 gene), } \\
\text { trancript variant } 2\end{array}$ \\
\hline 11 & $>$ gi|38149980 & $\begin{array}{l}\text { ref|NM_198220.1| Homo sapiens small nuclear ribonucleoprotein polypeptide B" (SNRPB2), transcript variant 2, } \\
\text { mRNA }\end{array}$ \\
\hline 12 & $>$ gi|71143122 & ref|NM_152551.3| Homo sapiens small nuclear ribonucleoprotein 48kDa (U11/U12) (SNRNP48), mRNA \\
\hline 13 & $>$ gi||164565439 & ref|NM_024571.3| Homo sapiens small nuclear ribonucleoprotein 25kDa (U11/U12) (SNRNP25), mRNA \\
\hline 14 & $>$ gi|38150006 & $\begin{array}{l}\text { ref|NM_198216.1| Homo sapiens small nuclear ribonucleoprotein polypeptides B and B1 (SNRPB), transcript } \\
\text { variant 1, mRNA }\end{array}$ \\
\hline 15 & $>\mathrm{gi} \mid 29540556$ & $\begin{array}{l}\text { ref|NM_003097.3| Homo sapiens small nuclear ribonucleoprotein polypeptide N (SNRPN), transcript variant 1, } \\
\text { mRNA }\end{array}$ \\
\hline 16 & $>$ gi|29540553 & $\begin{array}{l}\text { ref|NM_022806.2| Homo sapiens small nuclear ribonucleoprotein polypeptide N (SNRPN), transcript variant 3, } \\
\text { mRNA }\end{array}$ \\
\hline 17 & $>$ gi|50593001 & ref|NM_003090.2| Homo sapiens small nuclear ribonucleoprotein polypeptide A' (SNRPA1), mRNA \\
\hline 18 & $>$ gi|29294623 & $\begin{array}{l}\text { ref|NM_177542.1| Homo sapiens small nuclear ribonucleoprotein D2 polypeptide 16.5kDa (SNRPD2), transcript } \\
\text { variant 2, mRNA }\end{array}$ \\
\hline 19 & $>$ gi|29294622 & $\begin{array}{l}\text { ref|NM_004597.4| Homo sapiens small nuclear ribonucleoprotein D2 polypeptide 16.5kDa (SNRPD2), transcript } \\
\text { variant } 1 \text {, mRNA }\end{array}$ \\
\hline 20 & $>$ gi|28416941 & ref|NM_006938.2| Homo sapiens small nuclear ribonucleoprotein D1 polypeptide 16kDa (SNRPD1), mRNA \\
\hline 21 & $>$ gi|39725715 & ref|NM_004596.3| Homo sapiens small nuclear ribonucleoprotein polypeptide A (SNRPA), mRNA \\
\hline 22 & $>$ gi|83776586 & ref|NM_003095.2| Homo sapiens small nuclear ribonucleoprotein polypeptide F (SNRPF), mRNA \\
\hline 23 & $>\mathrm{gi} \mid 36497$ & emb|X52979.1| Human gene for small nuclear ribonucleoproteins SmB and SmB' \\
\hline 24 & $>$ gi|53690153 & gb|AY742712.1| Homo sapiens small nuclear ribonucleoprotein polypeptide B mRNA, complete cds \\
\hline 25 & $>$ gi|705365 & gb|AH003282.1|SEG_HUMSNRP0 Homo sapiens small nuclear ribonucleoprotein (SNRPD1) gene, complete cds \\
\hline 26 & $>$ gil|37539 & emb|X07401.1| Human mRNA for hU1-70K snRNP protein (RNP6) \\
\hline 27 & $>g i \mid 5870128$ & $\begin{array}{l}\text { gb|AH008174.1|SEG_HSSNRPB Homo sapiens small nuclear ribonucleoprotein (SNRPB) gene, alternative } \\
\text { splice products, complete cds }\end{array}$ \\
\hline 28 & $>$ gi|15217058 & $\begin{array}{l}\text { gb|AF400432.1|AF400432 Homo sapiens small nuclear ribonucleoprotein polypeptide N (SNRPN) mRNA, } \\
\text { complete cds }\end{array}$ \\
\hline 29 & $>$ gi|2708306 & gb|AF016370.1|AF016370 Homo sapiens U4/U6 small nuclear ribonucleoprotein hPrp3 mRNA, complete cds \\
\hline 30 & $>$ gi|2708304 & gb|AF016369.1|AF016369 Homo sapiens U4/U6 small nuclear ribonucleoprotein hPrp4 mRNA, complete cds \\
\hline 31 & $>\mathrm{gi} \mid 338249$ & gb|M21251.1|HUMSNRNP01 Human Alu repeats in the region 5' to the small nuclear ribonucleoprotein E gene \\
\hline 32 & $>g i \mid 37536$ & emb|X06818.1| Human mRNA for hU1-70K snRNP protein (RNPH2, part 1) \\
\hline 33 & $>g i \mid 37533$ & emb|X06817.1| Human mRNA for hU1-70k snRNP protein (RNPH1, part 1) \\
\hline 34 & $>$ gi|37532 & emb|X06816.1| Human mRNA for hU1-70K small nuclear RNP protein (RNP3) \\
\hline 35 & $>\operatorname{si|} 37530$ & emb|X06815.1| Human mRNA for hU1-70K small nuclear RNP protein (RNP FL1.7) (nonproductive) \\
\hline 36 & $>\mathrm{gi} \mid 37528$ & emb|X06814.1| Human mRNA for hU1-70K small nuclear RNP protein (RNP12) \\
\hline 37 & $>g i \mid 37524$ & emb|X06812.1| Human mRNA for hU1-70K snRNP protein (RNP8) \\
\hline 38 & $>$ gi|37522 & emb|X06811.1| Human mRNA for U1-70K snRNP protein (RNP11) \\
\hline 39 & $>\mathrm{gi} \mid 337442$ & gb|AH001532.1|SEG_HUMRNP70 Human small nuclear ribonucleoprotein (U1-70K) gene \\
\hline 40 & $>\mathrm{gi} \mid 337446$ & gb|M22636.1|HUMRNP70A Human U1 small nuclear ribonucleoprotein $70 \mathrm{kd}$ protein mRNA, complete cds \\
\hline
\end{tabular}


Table 2- Accession numbers and description of predominant types of Pseudoknots found in Human snurps

\begin{tabular}{|c|c|c|c|}
\hline Sl. No. & Accession No & Nt. Region & $\begin{array}{c}\text { Type of } \\
\text { pseudoknot }\end{array}$ \\
\hline 1.1 & >gi|40217846|ref|NM 014014.2| & $1-1711$ & None \\
\hline 1.2 & Do & $1712-1955$ & $\begin{array}{l}\text { HLIN-1 } \\
\text { HLout-1 }\end{array}$ \\
\hline 1.3 & Do & $1956-1276$ & HLout-1 \\
\hline 1.4 & Do & $1277-1560$ & HLOUT-1 \\
\hline 1.5 & Do & $1561-2979$ & None \\
\hline 1.6 & Do & 2980-3264 & HLout-1 \\
\hline 1.7 & Do & $3265-3547$ & HLout-1 \\
\hline 1.8 & Do & $3548-3827$ & LL-1 \\
\hline 1.9 & Do & $3828-4111$ & None \\
\hline 1.10 & Do & $4112-4394$ & $\begin{array}{l}\text { H type-2 } \\
\text { HLIN-1 }\end{array}$ \\
\hline 1.11 & Do & $4395-4675$ & None \\
\hline 1.12 & Do & 4676-4958 & LL-1 \\
\hline 1.13 & Do & $4959-5243$ & HLIN-1 \\
\hline 1.14 & Do & $5244-5528$ & $\begin{array}{l}\text { HLIN-1 } \\
\text { HLOUT-2 }\end{array}$ \\
\hline 1.15 & Do & $5529-6196$ & None \\
\hline 2.1 & >gi|31077202|ref|NM_022717.2| & $1-568$ & None \\
\hline 2.2 & Do & $569-997$ & HLIN-1 \\
\hline 3.1 & >gi|212549559|ref|NM_180703.2| & $1-285$ & HLout-1 \\
\hline 3.2 & Do & $286-1343$ & None \\
\hline 4.1 & >gi|57634537|ref|NM_003089.4| & $1-529$ & None \\
\hline 5.1 & >gi|38149990|ref|NM_003091.3| & $1-284$ & $\begin{array}{l}\mathrm{H}-1 \\
\mathrm{HL} \mathrm{IN}_{\mathrm{N}}-1\end{array}$ \\
\hline 6.1 & >gi|61098048|ref|NM_003094.2| & $1-284$ & HLout-1 \\
\hline 6.2 & Do & $285-566$ & $\begin{array}{l}\text { LL-1 } \\
\text { HLout-1 }\end{array}$ \\
\hline 6.3 & Do & $567-1134$ & None \\
\hline 6.4 & Do & $1135-1417$ & HLout-1 \\
\hline 6.5 & Do & $1418-1611$ & LL-1 \\
\hline 7.1 & >gi|29540552|ref|NM_022805.2| & $1-569$ & None \\
\hline 8.1 & >gi|29540554|ref|NM_022807.2| & $1-284$ & LL-1 \\
\hline 8.2 & Do & $285-565$ & None \\
\hline 8.3 & Do & $566-849$ & HLout-1 \\
\hline 8.4 & Do & $850-1420$ & None \\
\hline 8.5 & Do & $1421-1780$ & H type-1 \\
\hline 9.1 & >gi|4507126|ref|NM_003093.1| & $1-279$ & LL-1 \\
\hline 9.2 & Do & $280-564$ & HLIN-1 \\
\hline 9.3 & Do & $565-753$ & LL-1 \\
\hline 10.1 & >gi|24474790|emb|AJ505017.1| & $1-281$ & None \\
\hline 10.2 & Do & $282-566$ & HLout-1 \\
\hline 10.3 & Do & $567-847$ & $\mathrm{H}-1$ \\
\hline 10.4 & Do & $848-1844$ & None \\
\hline 10.5 & Do & $1845-2202$ & LL-1 \\
\hline 10.6 & Do & $2203-2951$ & None \\
\hline 11.1 & >gi|38149980|ref|NM_198220.1| & $1-353$ & LL-1 \\
\hline 11.2 & Do & $354-701$ & None \\
\hline 11.3 & Do & $702-986$ & $\begin{array}{l}\text { H-1 } \\
\text { HLout-1 }\end{array}$ \\
\hline 11.4 & Do & $987-1208$ & None \\
\hline 12.1 & >gi|71143122|ref|NM_152551.3| & $1-1063$ & None \\
\hline 12.2 & Do & $1064-1415$ & HLout-1 \\
\hline 12.3 & Do & $1416-1766$ & None \\
\hline 12.4 & Do & $1767-2119$ & LL-1 \\
\hline 12.5 & Do & $2120-3536$ & None \\
\hline 12.6 & Do & $3537-4188$ & HLout-1 \\
\hline
\end{tabular}




\begin{tabular}{|c|c|c|c|}
\hline 12.7 & Do & 4189-4494 & HLout-1 \\
\hline 13.1 & >gi|164565439|ref|NM_024571.3| & $1-358$ & LL-1 \\
\hline 13.2 & Do & $359-712$ & None \\
\hline 13.3 & Do & $713-1124$ & HLout-1 \\
\hline 14.1 & >gi|38150006|ref|NM_198216.1| & $1-354$ & LL-1 \\
\hline 14.2 & Do & $355-711$ & $\begin{array}{l}\mathrm{H}-1 \\
\mathrm{LL}-1\end{array}$ \\
\hline 14.3 & Do & $712-1036$ & LL-1 \\
\hline 15.1 & >gi|29540556|ref|NM_003097.3| & $1-354$ & LL-1 \\
\hline 15.2 & Do & $355-1715$ & None \\
\hline 16.1 & >gi|29540553|ref|NM_022806.2| & $1-1044$ & None \\
\hline 16.2 & Do & $1045-1399$ & $\mathrm{H}-1$ \\
\hline 16.3 & Do & $1400-1626$ & None \\
\hline 17.1 & >gi|50593001|ref|NM_003090.2| & $1-357$ & None \\
\hline 17.2 & Do & $358-711$ & HLout-1 \\
\hline 17.3 & Do & $712-1088$ & None \\
\hline 18.1 & >gi|29294623|ref|NM_177542.1| & $1-354$ & HLout-1 \\
\hline 18.2 & Do & $355-707$ & HLout-1 \\
\hline 18.3 & Do & $708-804$ & None \\
\hline 19.1 & >gi|29294622|ref|NM_004597.4| & $1-425$ & LL-1 \\
\hline 19.2 & Do & $426-790$ & HLout-1 \\
\hline 20.1 & >gi|28416941|ref|NM_006938.2| & $1-711$ & None \\
\hline 20.2 & Do & $712-1066$ & HLout-1 \\
\hline 20.3 & Do & $1167-1633$ & None \\
\hline 21.1 & >gi|39725715|ref|NM_004596.3| & $1-780$ & None \\
\hline 21.2 & Do & $781-1133$ & LL-1 \\
\hline 21.3 & Do & $1134-1646$ & None \\
\hline 22.1 & >gi|83776586|ref|NM_003095.2| & $1-427$ & $\mathrm{H}-1$ \\
\hline 22.2 & Do & $428-824$ & None \\
\hline 23.1 & >gi|36497|emb|X52979.1| & $1-423$ & HLout-1 \\
\hline 23.2 & Do & $424-959$ & None \\
\hline 24.1 & $>g i|53690153| g b|A Y 742712.1|$ & $1-355$ & HLout-1 \\
\hline 24.2 & Do & $356-717$ & None \\
\hline 25.1 & $>$ gi|705365|gb|AH003282.1| & $1-427$ & None \\
\hline 25.2 & Do & $428-855$ & $\begin{array}{l}\text { HLIN-1 } \\
\text { HLOUT-1 }\end{array}$ \\
\hline 25.3 & Do & $856-2131$ & None \\
\hline 25.4 & Do & $2132-2548$ & HLout-1 \\
\hline 26.1 & >gi|37539|emb|X07401.1| & $1-717$ & None \\
\hline 26.2 & Do & $718-1027$ & HLout-1 \\
\hline 27.1 & $>$ >gi|5870128|gb|AH008174.1| & $1-423$ & $\begin{array}{l}\text { H-1 } \\
\text { HLout-1 }\end{array}$ \\
\hline 27.2 & Do & $424-1717$ & None \\
\hline 27.3 & Do & $1718-2145$ & HLout-1 \\
\hline 27.4 & Do & $2146-3857$ & None \\
\hline 27.5 & Do & $3858-4285$ & HLOUT-1 \\
\hline 27.6 & Do & $4286-5137$ & None \\
\hline 27.7 & Do & $5138-5510$ & $\mathrm{H}-1$ \\
\hline 28.1 & $\begin{array}{l}>\text { gi|15217058|gb|AF400432.1|AF } \\
400432\end{array}$ & $1-835$ & None \\
\hline 29.1 & $\begin{array}{l}>\text { gi|2708306|gb|AF016370.1|AF0 } \\
16370\end{array}$ & $1-428$ & $\mathrm{H}-1$ \\
\hline 29.2 & Do & $429-1271$ & None \\
\hline 30.1 & $\begin{array}{l}>\text { gi|2708304|gb|AF016369.1|AF0 } \\
16369\end{array}$ & $1-1259$ & None \\
\hline 30.2 & Do & $1260-1683$ & HLout-1 \\
\hline 30.3 & Do & $1684-2188$ & None \\
\hline 31.1 & $\begin{array}{l}>\text { gi|338249|gb|M21251.1|HUMSN } \\
\text { RNP01 }\end{array}$ & $1-426$ & HLout-1 \\
\hline 31.2 & Do & $427-758$ & None \\
\hline 32.1 & >gi|37536|emb|X06818.1| & $1-140$ & None \\
\hline 33.1 & >gi|37533|emb|X06817.1| & $1-257$ & None \\
\hline 34.1 & $>$ gi|37532|emb|X06816.1| & $1-531$ & $\begin{array}{l}\text { H-1 } \\
\text { HLout-1 }\end{array}$ \\
\hline
\end{tabular}




\begin{tabular}{|c|c|c|c|}
\hline 35.1 & $>$ gi|37530|emb|X06815.1| & $1-851$ & None \\
\hline 35.2 & Do & $852-1184$ & HLout-1 \\
\hline 35.3 & & $1185-1593$ & None \\
\hline 36.1 & $>$ gi|37528|emb|X06814.1| & $1-358$ & None \\
\hline 36.2 & Do & $359-718$ & HLIN-1 \\
\hline 37.1 & $>$ gi|37524|emb|X06812.1| & $1-423$ & LL-1 \\
\hline 37.2 & Do & $422-710$ & None \\
\hline 37.3 & Do & $711-918$ & HLout-1 \\
\hline 38.1 & $>$ gi|37522|emb|X06811.1| & $1-400$ & LL-1 \\
\hline 39.1 & $\begin{array}{l}\text { >gi|337442|gb|AH001532.1|SEG } \\
\text { HUMRNP70 }\end{array}$ & $1-429$ & None \\
\hline 39.2 & Do & $430-858$ & $\begin{array}{l}\mathrm{H}-1 \\
\mathrm{H} L_{I_{N}-1}\end{array}$ \\
\hline 39.3 & Do & $859-1283$ & None \\
\hline 39.4 & Do & $1284-1639$ & LL-1 \\
\hline 39.5 & Do & $1640-2063$ & LL-1 \\
\hline 39.6 & Do & $2064-2417$ & None \\
\hline 39.7 & Do & $2418-2772$ & HLout-1 \\
\hline 39.8 & Do & $2773-3836$ & None \\
\hline 39.9 & Do & $3486-3837$ & HLout-1 \\
\hline 39.10 & Do & $3838-4194$ & HLout-1 \\
\hline 39.11 & Do & $4195-4556$ & HLout-1 \\
\hline 39.12 & Do & $4557-4985$ & HLout-1 \\
\hline 39.13 & Do & $4986-6267$ & None \\
\hline 39.14 & Do & $6268-6667$ & $\mathrm{HL}_{\mathrm{IN}}-1$ \\
\hline 40.1 & $>$ gi|337446|gb|M22636.1| & $1-496$ & HLout-1 \\
\hline 40.2 & Do & 497-995 & None \\
\hline 40.3 & Do & $996-1358$ & HLIN-1 \\
\hline 40.4 & Do & $1359-1690$ & None \\
\hline
\end{tabular}

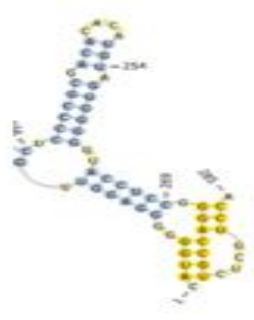

(a)HL ort type

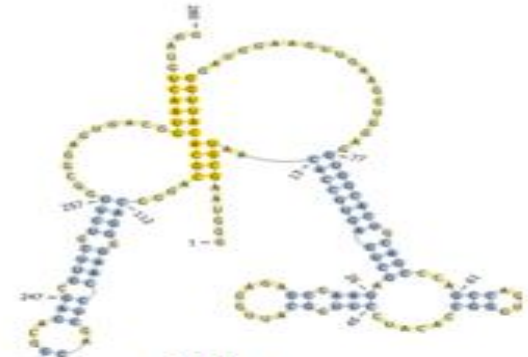

(b) II type



(c) HIax Type



(d) $\mathrm{H}$ type

Fig. 1 (a-d): Four major types of pseudoknots found in Human snRNPs. 